\title{
Correction to: Optimal Control of a Phase Field System Modelling Tumor Growth with Chemotaxis and Singular Potentials
}

\author{
Pierluigi Colli ${ }^{1}$. Andrea Signori ${ }^{1}$ (i) $\cdot$ Jürgen Sprekels ${ }^{2,3}$ \\ Published online: 15 April 2021 \\ (c) Springer Science+Business Media, LLC, part of Springer Nature 2021
}

\section{Correction to: Applied Mathematics \& Optimization https://doi.org/10.1007/s00245-019-09618-6}

We list the corrections that have to be carried out in the paper.

- Three lines above the formula $(2.1)$, the space $L^{2}(Q)$ should be replaced by $L^{2}(Q)^{2}$.

- In the definition (2.1) of the set $\mathcal{U}_{R}$, the space and norm of $L^{2}(Q) \times L^{2}(Q)$ should be replaced by $L^{\infty}(Q) \times L^{\infty}(Q)$. Namely, the new formula (2.1) should be

$$
\mathcal{U}_{R}:=\left\{(u, w) \in L^{\infty}(Q) \times L^{\infty}(Q):\|(u, w)\|_{L^{\infty}(Q) \times L^{\infty}(Q)}<R\right\} \text { contains } \mathcal{U}_{\mathrm{ad}} .
$$

- In Theorem 2.3 the assumptions (A1)-(A7) should be replaced by (A1)-(A4).

- In Theorem 2.6 the assumption (A7) is not needed and the space $\left(L^{2}(Q)\right)^{2}$ has to be changed in $\left(L^{\infty}(Q)\right)^{2}$, twice.

- In the proof of Theorem 2.2 the Sixth estimate, involving the formulae (3.11) and (3.12), has to be moved immediately after formula (3.5) and before the current Fourth estimate.

The original article can be found online at https://doi.org/10.1007/s00245-019-09618-6.

Andrea Signori

andrea.signori02@universitadipavia.it

Pierluigi Colli

pierluigi.colli@unipv.it

Jürgen Sprekels

sprekels@wias-berlin.de

1 Dipartimento di Matematica “F. Casorati”, Università di Pavia, via Ferrata 5, 27100 Pavia, Italy

2 Department of Mathematics, Humboldt-Univesität zu Berlin, Unter den Linden 6, 10099 Berlin, Germany

3 Weierstrass Institute for Applied Analysis and Stochastics, Mohrenstrasse 39, 10117 Berlin, Germany 
- At the beginning of the proof of Theorem 2.3, one should completely remove the three lines below (3.18) "In view of ... (1.1)-(1.5)".

- In the formula preceding (3.21), in the last line, the term $\left\|u^{*}\right\|_{L^{\infty}(Q)}^{2}$ has to be replaced by $\left\|u_{2}\right\|_{L^{\infty}(Q)}^{2}$. Moreover, the lines below should be changed into "where we use the boundedness of $\sigma_{2}$ once more. Thus, the above estimates yield that".

- In the proof of Theorem 2.6, the initial part should be changed into:

"At first, let us fix a control pair $(\bar{u}, \bar{w}) \in \mathcal{U}_{\text {ad }} \subset \mathcal{U}_{R}$ with the corresponding state $(\bar{\mu}, \bar{\varphi}, \bar{\sigma})$. Then, whenever $(k, l)$ belongs to $\left(L^{\infty}(Q)\right)^{2}$, we denote with $(\eta, \xi, \zeta)$ the corresponding solution to system (2.8)-(2.12). Moreover, let us recall that $\mathcal{U}_{R}$ is an open set, so that, provided that we consider small perturbations, we also have $(\bar{u}+k, \bar{w}+l) \in \mathcal{U}_{R}$. Namely, there exist some positive constant $\delta_{*}$ such that $(\bar{u}+k, \bar{w}+l) \in \mathcal{U}_{R}$ for every $(k, l)$ such that $\|k\|_{L^{\infty}(Q)}+\|l\|_{L^{\infty}(Q)} \leq \delta_{*} . "$

- In the proof of Theorem 2.6, starting from formula (4.10) to formula (4.11), in all the occurrences the space $\left(L^{2}(Q)\right)^{2}$ and the respective norm $\|\cdot\|_{L^{2}(Q) \times L^{2}(Q)}$ have to be substituted by $\left(L^{\infty}(Q)\right)^{2}$ and $\|\cdot\|_{L^{\infty}(Q) \times L^{\infty}(Q)}$, respectively.

- In the proof of Theorem 2.6, in the estimate of term $\left|I_{2}\right|,\left\|u^{*}\right\|_{L^{\infty}(Q)}$ has to be replaced by $\|\bar{u}\|_{L^{\infty}(Q)}$, three times.

- As a remark, let us point out that, about the end of the proof of Theorem 2.6, the last inequality does provide the claim (4.11). In fact it suffices to employ the continuous embedding $L^{\infty}(Q) \times L^{\infty}(Q) \subset L^{2}(Q) \times L^{2}(Q)$ to conclude.

Publisher's Note Springer Nature remains neutral with regard to jurisdictional claims in published maps and institutional affiliations. 